\title{
Gottwald Melborune (0-1) test for chaos in a plasma
}

\author{
D. R. Chowdhury ${ }^{1}$, A. N. S. Iyengar ${ }^{2}$, and S. Lahiri ${ }^{3}$ \\ ${ }^{1}$ Techno India, EM4/1, Sector V, Kolkata-700091, India \\ ${ }^{2}$ Saha Institute of Nuclear Physics, 1/AF Bidhan Nagar, Kolkata-700064, India \\ ${ }^{3}$ Dinabandhu Mahavidyalaya, Bongaon, North 24 Parganas, Kolkata-743235, India \\ Correspondence to: A. N. S. Iyengar (ansekar.iyengar@saha.ac.in)
}

Received: 11 November 2010 - Revised: 6 June 2011 - Accepted: 15 October 2011 - Published: 10 January 2012

\begin{abstract}
Plasma is a highly complex system exhibiting a rich variety of nonlinear dynamical phenomena. In the last two decades or so there has been a spurt of growth in exploring unconventional nonlinear dynamical methods of analysis, like chaos theory, multi fractal analysis, self organized criticality etc. of experimental data from different plasma systems. Investigation of fluctuating plasma parameters is very important since they are correlated with transport of particles, and energy. In time series analysis, it is considered of key importance to determine whether the data measured from the system is regular, deterministically chaotic, or random. The two important parameters that are in general estimated are the correlation dimension and the Lyapunov exponent. Though correlation dimension helps in determining the complexity of a system, Lyapunov exponent reveals if the system is chaotic or not and also helps in prediction to some extent. In spite of its extensive usage, estimation of Lyapunov exponent can be quite tedious and sometimes suffers from some disadvantages like reliability in the presence of noise, requirement of phase space reconstruction etc., and hence it is necessary to explore other possibilities of estimating the chaoticity of a data. In this paper we have analysed for chaoticity, the nonlinear floating potential fluctuations from a glow discharge plasma system by the $0-1$ test and compared it with the results obtained from Lyapunov exponent.
\end{abstract}

\section{Introduction}

Chaos theory which emerged as a subsection of nonlinear dynamics has gained a significant importance in the last two to three decades especially since it could explain several of the experimental observations not only in physics but also in other areas like biology, environmental sciences etc. Chaotic systems may seem random, and unpredictable, but it has been shown that they can be modeled by equations that can be formulated without much difficulty. Ever since the first experi- mental observation of chaos in a large magnetized plasma device (Cheung and Wong, 1987) many experiments have been carried out in this area in different plasma systems (Ding et al., 1993; Lin and Jeng-Mei, 1995; Klinger et al., 1995; Dinklage et al., 1999; Jaman and Iyengar, 2007; Jaman et al., 2008).

Plasma systems being inherently a complex medium can exhibit a wide range of behaviour from chaos to order and vice versa by changing a parameter externally. In many of the experiments, order to chaos has been verified from power spectral analysis only and in some cases only by estimation of the correlation dimension without verifying through Lyapunov exponents. In any experimental data it is always wise to cross check the results by as many techniques as possible since it increases the confidence level of the techniques and also the experimental results. Gottwald and Melbourne (2004) proposed a new test called the $0-1$ test to determine if the time series was of chaotic nature or not, wherein a 0 implied regular and a 1 implied a chaotic series. Some of the advantages of this test over the estimation of the largest Lyapunov exponent are that (a) it does not require phase space reconstruction, (b) the dimension of the dynamical system does not pose practical limitations on the method as is the case for traditional methods involving phase-space reconstruction, (c) the form of the underlying equations are irrelevant, (d) the input is the time-series data and the output is 0 or 1 , depending on whether the dynamics is non chaotic or chaotic, and (e) the test is universally applicable to any deterministic dynamical system. In this paper we have carried out a detailed analysis of the chaotic fluctuations of floating potential in a glow discharge plasma using the power spectrum, Lyapunov exponents and the $0-1$ test. The Largest Lyapunov exponent was estimated by Rosenstein's method (Rosenstein et al., 1993) which can be applied to short data sets. In Sect. 2 we briefly describe the methodology of the $0-1$ test followed by Results and Discussion in Sect. 3 and conclusions in Sect. 4. 


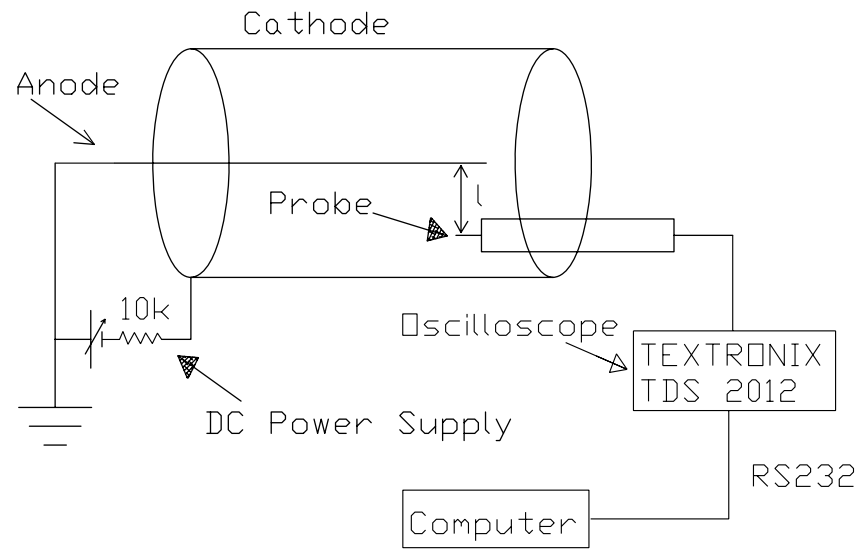

Fig. 1. Schematic diagram of the hollow cathode discharge tube. The cathode and the anode are a hollow tube and a wire of stainless steel, respectively.

\section{Methodology}

The $0-1$ test was initially proposed by Gottwald and Melbourne (2004) which was subsequently modified and made simpler (Gottwald and Melbourne, 2005). This test yields a binary type of result: 0 for regular (periodic/quasi periodic) and 1 for chaotic signals. If $x(n)$ is an observable in time, we can define dimensionless displacements in the $(p, q)$ plane as follows:

$p(n)=\sum_{j=1}^{N} \times(n) \cos (j c)$

and

$q(n)=\sum_{j=1}^{N} \times(n) \cos (j c)$

where $c$ is a constant chosen at random between $(0,2 \pi)$. If the system dynamics is chaotic, the motion in the $(p, q)$ plane would be unbounded, while it would be bounded for regular (periodic/quasiperiodic) motion. The mean square displacement is expressed as

$$
\begin{aligned}
M(n)= & \lim _{N \rightarrow \infty}(1 / N) \sum_{j=1}^{N}[p(j+n)-p(n)]^{2} \\
& +[q(j+n)-q(n)]^{2}, n=1,2, . .
\end{aligned}
$$

For regular dynamics (periodic or quasi periodic), it is quite likely that $M(n)$ will be a bounded function of $n$ but not for chaotic dynamics. From this one can define the asymptotic growth rate of the mean square displacement $k$

$k=\lim _{n \rightarrow \infty} \frac{\log M(n)}{\log (n)}$

for which $k=0$ signifies regularity while $k=1$ indicates presence of chaoticity. Since we had estimated $k$ for about
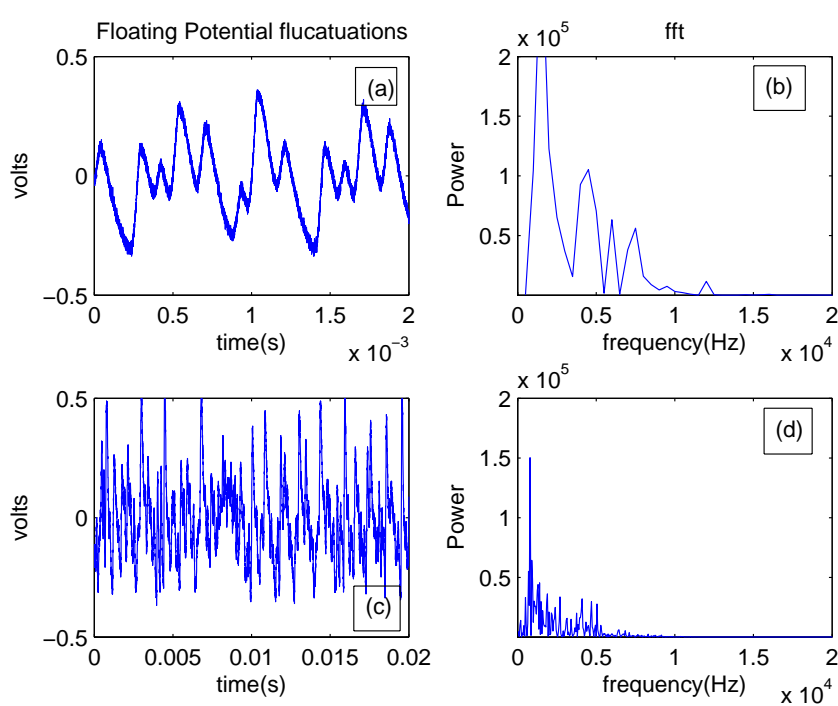

Fig. 2. Top plots are the quasi periodic data (a) and its $\mathrm{fft}(\mathbf{b})$, while the bottom plots are the chaotic data (c) and its fft (d).

hundred values of $c$, the median of $k$ was estimated and if it was very small $(<0.05)$ we concluded that the system dynamics was regular and chaotic if it was $\simeq 1$.

\section{Results and discussion}

The experiments were performed in a hollow cathode glow discharge plasma similar to the one used in the earlier experiments by Jaman and Iyengar (2007) as shown in Fig. 1, but with an increased diameter by a factor of 2 . The plasma floating potential fluctuations were monitored by a Langmuir probe with a sampling frequency of about $2 \mathrm{MHz}$ for Fig. $2 \mathrm{a}$ and $500 \mathrm{kHz}$ for Fig. 2b, respectively.

Keeping the gas filling pressure constant, we varied the discharge voltage until we could ionize the neutral gas. At this instant, the fluctuating component of the floating potential monitored by a Langmuir probe was initially quasi periodic and with increase in discharge voltage it continued to remain so as in Fig. 2a, until a threshold value of discharge voltage, beyond which it became chaotic as seen in Fig. 2c. The difference between the two cases was that in the quasi periodic case there was a faint reddish plasma around the anode which changed to a bluish glow confined to the cathode region for the chaotic one. The fft (fast fourier transform) of the quasi periodic oscillations shows a large peak at about $7.5 \mathrm{kHz}$ and some distinct frequencies of reduced amplitudes, whereas for the chaotic case there is a clear signature of turbulence seen in the up to about $5 \mathrm{kHz}$ and with much reduced amplitude beyond, which not shown here. The largest Lyapunov exponent estimated by Rosenstein's (1994) method for the quasi periodic case was about 0.002 for Fig. 2a which increased to 0.02 for Fig. 2c, as seen 


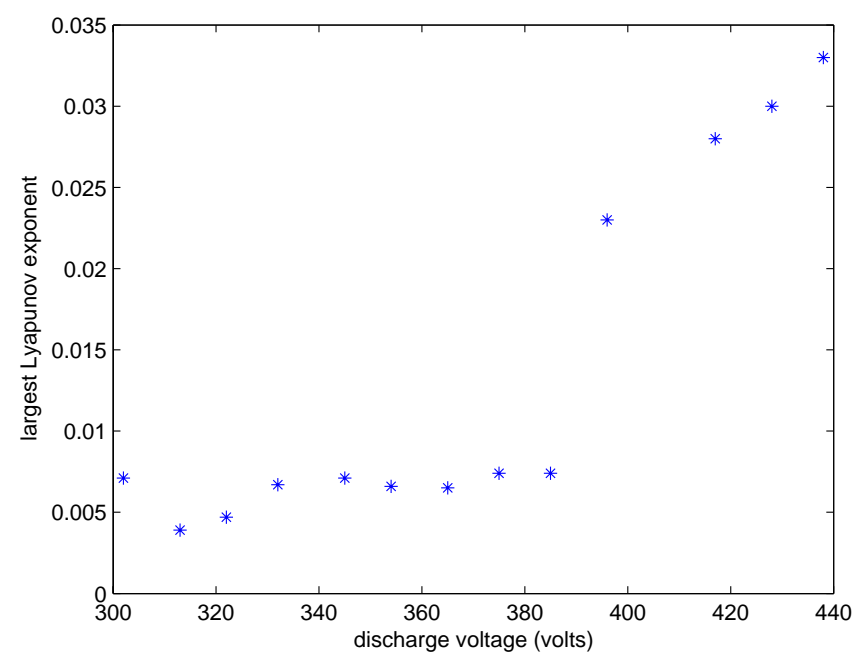

Fig. 3. Largest Lyapunov exponent shows a clear jump when the floating potential fluctuations exhibiting a transition from quasi periodic to chaos.
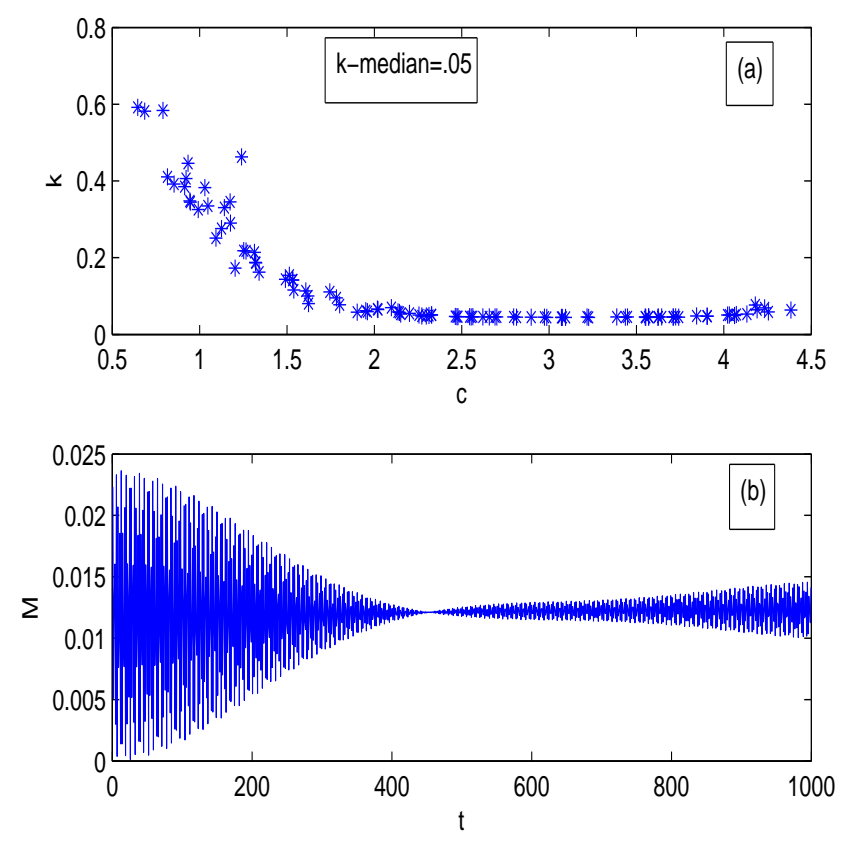

Fig. 4. (a) corresponds to the value of $k$ as a function of $c$, with the median value shown in the box, and (b) is a plot of $M(n)$ which shows a constant value and hence the slope is zero.

in Fig. 3. Though it is positive for the quasiperiodic and the chaotic signals, it is very small for the former and shows an increase by a factor of 10 for the latter indicating the clear presence of chaoticity.

Next we carried out the 0-1 test (Gottwald and Melbourne, 2004) after filtering out the frequencies above
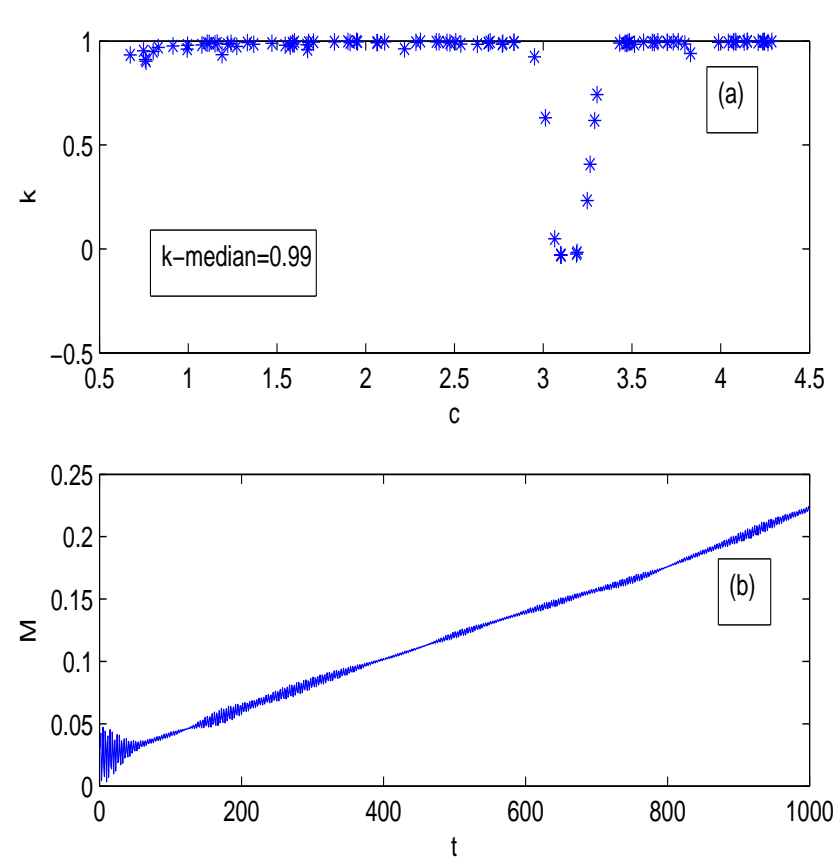

Fig. 5. (a) corresponds to the value of $k$ as a function of $c$, with the median value shown in the box, and (b) is a plot of $M(n)$ which shows an increasing value with $n$ and hence a positive slope confirming the presence of chaos.

$200 \mathrm{kHz}$ for both the data sets, choosing at random, about 100 values of $c$ ranging from $\pi / 5$ to $6 \times \pi / 5$. Figure 4 and Fig. 5 show the graphs of $k$ vs $c$ and $M(n)$ vs $n$ for the quasi periodic and chaotic data, respectively. Figure 4a shows a significant portion where $k$ is very low of the order of 0.04 , and the $M(n)$ vs $n$ also shows almost a zero slope. For the chaotic data $k$ vs $c$ is around 1 , while $M(n)$ vs $n$ is showing a positive slope.

$k$-median for the quasi periodic was about 0.05 for the quasi periodic while it was about 0.9 for the chaotic case. From this we can concluded that this is a very efficient test for chaos in an experimental time series. There may be some difficulties since almost experimental results have in them some noise that one may have to filter out. The other precaution that one has to take is probably the over sampling. We are in the process of carrying out a detailed investigation of the validity of 0-1 test taking into account features like over sampling, filtering, surrogates etc which are a part of our future plans. 


\section{Conclusions}

We have applied the $0-1$ test to the time series obtained from a glow discharge plasma experiment, and it looks to be very effective and simpler than the estimation of the largest Lyapunov exponent since we are working directly on the time series and also there is less ambiguity that may arise in the estimation of the largest Lyapunov exponent. We will be carrying out a detailed analysis of the same for various types of filtering, over sampling etc as a part of our future plans.

Acknowledgements. We would like to thank the members of plasma physics division for the experimental support and the Director, SINP, for the encouragement provided.

Edited by: A. Bunde

Reviewed by: H. Chaudhuri and another anonymous referee

\section{References}

Cheung, P. Y. and Wong, A. Y.: Chaotic behaviour and periodic doubling in plasmas, Phys. Rev. Lett., 59, 551-554, 1987.

Ding Weixing, Huang Wei, Wang Xiaodong, and C. X. Yu: Chaos in glow discharge plasma, Phys. Rev. Lett. 70, 170-173,1993.

Dinklage, A., Wilke, C., and Klinger, T.: Spatiotemporal response of stochastic resonance in an excitable discharge plasma, Phys. Plasmas, 6, 2968-2971, 1999.

Gottwald, G. A. and Melbourne, I.: A new test for chaos in a deterministic system, Proc. Roy. Soc. A, 460, 603-611, 2004.

Gottwald, G. A. and Melbourne, I.: Testing for chaos in a deterministic system with noise Physica D, 460, 212, 100-110, 2005.

Klinger, T., Greiner, T., Rohde, A., Piel, A., and Koepke, M. E.: Van der Pol behaviour of relaxation oscillations in a periodically driven thermionic discharge, Phys. Rev. E, 52, 4316-4327, 1995.

Lin, I. and Jeng-Mei, L.: Experimental observation of stochastic resonance like behaviour of autonomous motion in weakly ionized rf magnetoplasmas, Phys. Rev. Lett., 74, 3161-3164, 1995.

Nurujjaman, M. D. and Sekar Iyengar, A. N.: Realization of SOC behaviour in a dc glow discharge plasma, Phys. Lett. A, 360, 717-721, 2007.

Nurujjaman, M. D., Sekar Iyengar, A. N., and Parmananda, P.: Noise invoked resonances near homoclinic bifurcation in the glow discharge plasma, Phys. Rev. E, 78, 026406-1-026406-5, 2008.

Rosenstein, M. T., Collins, J. J., and De Luca, C. J.: A practical method for calculating largest Lyapunov exponent for small data sets, Physica D, 65, 117-134, 1993. 